\title{
Analysis of Correlation Between Whole Exome Sequencing and Ultrasound Examinationin Prenatal Diagnosis of Fetal Skeletal Dysplasia
}

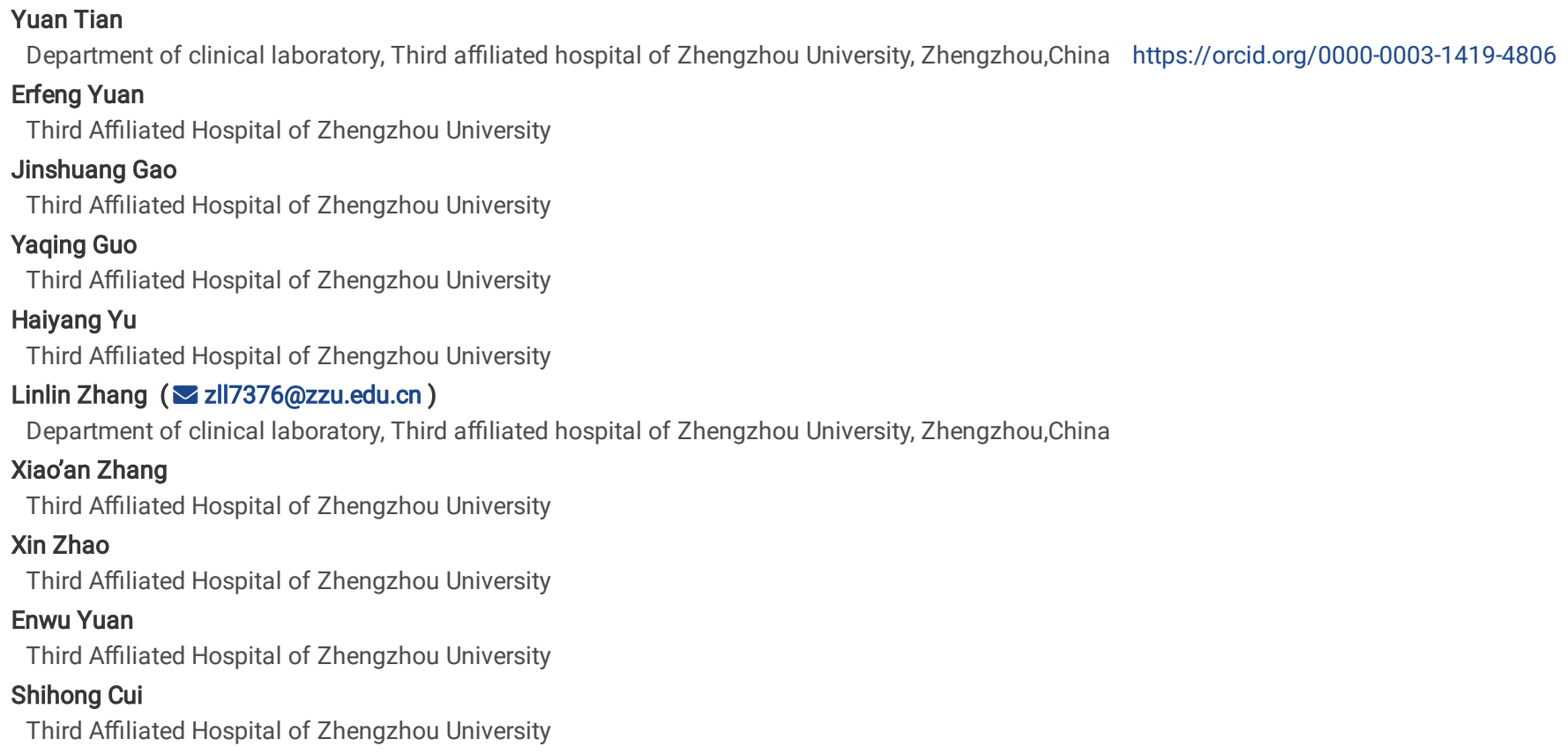




\section{Abstract}

Background:Fetal skeletal dysplasia is a disease that is difficult to distinguish these types of diseases during the fetal period. Due to the difficulty of fetal ultrasound diagnosis, the severity of fetal skeletal dysplasia is extremely difficult to assess.

Methods: 79 fetal samples of skeletal dysplasia from the third affiliated hospital of Zhengzhou University, China from August 2018 to April 2020 , which had undergone prenatal whole exome sequencing(WES) were analyzed and the results of whole-exome sequencing and fetal ultrasound test results were compared.

Results:We find that the fetal short limb phenotype found in the range of $\mathrm{FL}<-4.0 \mathrm{SD}$ or $\mathrm{HL}<-4.0 \mathrm{SD}$ through ultrasound test is closely related to FGFR3 gene mutation, and the correlation is stronger when accompanied by macrocephaly. We also find that the fetal limb curved phenotype is closely related to $C O L 1 A 1$ gene mutation. At the same time, we find that nasal dysplasia during fetal period is also a common phenotype of abnormal results detected by whole exome sequencing.

Conclusions: Our research shows that WES has different detection rates for various skeletal abnormalities according to the different types of ultrasound detection results, which provides a meaningful guidance for clinical diagnosis of fetal skeletal dysplasia.

\section{Introduction}

Fetal skeletal dysplasia is a disease of osteochondrocytes with strong clinical variability[1,2]. Due to their rarity, it is difficult to distinguish these types of diseases during the fetal period[3]. The diagnosis of fetal skeletal dysplasia can be done through prenatal ultrasound evaluation[3]. Skeletal dysplasia often causes a reduction in bust size and leads to lung dysplasia, so fetal skeletal dysplasia is often fatal. However, due to the difficulty of fetal ultrasound diagnosis, the severity of fetal skeletal dysplasia is extremely difficult to assess[4]. The accuracy of conventional ultrasound on fetal skeletal dysplasia is no more than $40 \%[4]$. Misdiagnosis can lead to erroneous information about the risk of relapse and delay optimal fetal management.

Using fetal imaging as a guide, a personalized prenatal genetic examination strategy can be selected. Current options include chromosome karyotype analysis, chromosome fluorescence in situ hybridization experiments, chromosome microarray analysis, whole exome sequencing etc[5]. According to the 2010 revision of the Nosology and Classification of Genetic Skeletal Disorders, 456 diseases were divided into 40 groups according to molecular, biochemical and radiological standards[6]. Among them, 316 bone-related diseases are related to the mutation of one or more genes in 226 different genes, which provides a basis for the molecular genetic diagnosis of fetal skeletal development abnormalities[6]. Two recent studies have shown that in the assessment of large scale prospectively ascertained cohort of fetal with skeletal anomalies, the diagnostic rates of skeletal dysplasia are 10/65 (15.4\%)[7] and 8/34 (24\%)[5], respectively.

In our study, we analyzed 79 fetal samples of skeletal dysplasia from the third affiliated hospital of Zhengzhou University, China from August 2018 to April 2020, which had undergone prenatal whole exome sequencing(WES). Our research aims to explore the genetic types of fetal skeletal dysplasia in China, and explore the correlation between results by WES and phenotype of skeletal dysplasia in the fetal period by ultrasound, with a view to providing a theoretical basis for early implementation of birth defect intervention and reproductive risk assessment.

\section{Results}

\section{Correlation between positive results by WES and clinical phenotypes by ultrasound}

Among 79 pregnant women from the third affiliated hospital of Zhengzhou University, China, from August 2018 to April 2020,25 cases that were positive by WES were detected, whose detection rate was $31.6 \%$.Among these cases, the detection rate of cases with only $\mathrm{FL}<-4.0 \mathrm{SD}$ or only $\mathrm{HL}<-4.0 \mathrm{SD}$ was $41.6 \%$ (5/12). The detection rate of cases with both $\mathrm{FL}<-4.0 \mathrm{SD}$ and $\mathrm{HL}<-4.0 \mathrm{SD}$ is $60 \%(3 / 5)$. In cases of $-4.0 \mathrm{SD}<\mathrm{FL}<-2.0 \mathrm{SD}$ or $-4.0 \mathrm{SD}<\mathrm{HL}<-2.0 \mathrm{SD}$, the detection rate by WES was $29.4 \%$ (5/17). However,in these cases where exsists $-4.0 \mathrm{SD}<\mathrm{FL}<-2.0 \mathrm{SD}$ or $-4.0 \mathrm{SD}<\mathrm{HL}<-2.0 \mathrm{SD}$, with long bone bending, the detection rate by WES was $100 \%$ $(2 / 2)$. In cases of $\mathrm{FL}<-2.0 S D$ or $\mathrm{HL}<-2.0 S D$, the detection rate by WES is $20 \%(1 / 5)$, where the only one positive case besides $\mathrm{FL}<-2.0 \mathrm{SD}$ and $\mathrm{HL}<-2.0 \mathrm{SD}$, there exsists also femoral curvature. In cases with only long bone bending, the detection rate by WES is $50 \%(2 / 4)$.In cases of nasal bone dysplasia, the detection rate by WES is $80 \%(4 / 5)$.It is worth mentioning that cases of only microcephaly have not been detected positive by WES. Only 1 of 9 cases of fetal hand or foot deformity were positive by WES (11.1\%).(Table .1)

\section{Correlation between abnormal results of FGFR3 gene sequenced by WES and clinical phenotypes by ultrasound}

Among 25 cases that are positive by WES, 7 cases are caused by FGFR3 gene mutation, accounting for 28\%.Among them, 6 cases are FGFR3 gene c. $1138 \mathrm{G}>\mathrm{A}$ mutation, and 1 case is FGFR3 gene c.1620C>A mutation.In these 7 cases, the ultrasound test results of almost all cases show $\mathrm{FL}<-4.0 \mathrm{SD}$ or $\mathrm{HL}<-4.0 \mathrm{SD}(\mathrm{Only}$ one case of short limbs with ambiguous data),among which 3 cases of ultrasound test results also show macrocephaly, accounting for $42.8 \%$.Besides, all genetic variations are de novo.(Table.2)

\section{Correlation between abnormal results of COL1A1 gene sequenced by WES and clinical phenotypes by ultrasound}

Among 25 cases that are positive by WES, 3 cases are caused by COL $1 A 1$ gene mutation, accounting for $12 \%$. In these 3 cases, the ultrasound test results of 2 cases show FL>-4.0SD or HL>-4.0SD, ), among which all of 3 cases of ultrasound test results showfetal limbs slightly curved, accounting for $100 \%$. Besides, One of the three cases has a COL1A1 gene variant inherited from the pregnant husband, and the rest of COL1A1 gene variant are de novo. (Table.3)

Correlation between abnormal results of other gene sequenced by WES and clinical phenotypes by ultrasound 
Among 25 cases that are positive by WES, 11 cases are caused by other gene mutation accounting for $44 \%$. In these 11 cases,there are 2 cases caused by RUNX2 gene mutation, both of which have nasal bones not shown by ultrasound.In addition, there are 2 cases due to COL2A1 gene mutation, both of which have short limbs by ultrasound.The other 7 cases are caused by ARSE gene mutation, EFTUD2 gene mutation, SCN4A gene mutation, COL $1 A 2$ gene mutation, $P E X 7$ gene mutation, NEB gene mutation and FGFR2 gene mutation. Ultrasound test results related to these 7 genetic variations are mainly manifested by phenotypes other than short limbs, such as microcephaly, bilateral foot varus, the femur is slightly curved, abnormal ossification of both humerus and femur, fingers merge, toes merge, etc.(Table.4)

\section{Correlation between abnormal results of copy number variation (CNV) sequenced by WES and clinical phenotypes by ultrasound}

Among 25 cases that are positive by WES,4cases are caused by copy number variation accounting for $16 \%$.Among these 4 cases, 3 cases were caused by chromosome copy number variation, and 1 case was chromosomal aneuploidy (Trisomy 21). 3 cases of chromosomal copy number variation were all deletions, and their phenotypes by ultrasound varied. 1 case of trisomy 21 has a phenotype for nasal bone dysplasia by ultrasound.(Table.5)

Table.1 Correlation between positive results by WES and clinical phenotypes by ultrasound 


\begin{tabular}{|c|c|c|c|c|}
\hline ID & Age(years) & $\begin{array}{l}\text { Gestational } \\
\text { age(weeks) }\end{array}$ & Phenotype by ultrasound & $\begin{array}{l}\text { Result } \\
\text { by WES }\end{array}$ \\
\hline 1 & 31 & $22^{+2}$ & Fetal vertebral fusion & Negative \\
\hline 2 & 28 & 25 & $\mathrm{HL}<-3.2 \mathrm{SD}, \mathrm{FL}<-2.1 \mathrm{SD}$; both femurs are slightly curved & Positive \\
\hline 3 & 28 & 23 & Fetal punctate achondroplasia & Negative \\
\hline 4 & 35 & 26 & Fetal Multi-finger & Negative \\
\hline 5 & 25 & 24 & Curved fetal osteogenesis & Positive \\
\hline 6 & 26 & 21 & $\begin{array}{l}\text { Poor skull development, nasal bones not shown, poor ossification of the lumbar vertebral body of the } \\
\text { spine, poor ossification of the sacral caudal vertebral arch and vertebral body }\end{array}$ & Positive \\
\hline 7 & 32 & 33 & $\mathrm{HL}<-5.9 \mathrm{SD}, \mathrm{FL}<-5.9 \mathrm{SD} \bigotimes$ metaphyseal enlargement & Positive \\
\hline 8 & 30 & 21 & Right femur slightly curved & Negative \\
\hline 9 & 30 & 24 & $\begin{array}{l}\text { The long bones of the limbs are obviously short, the alveolar bone is flat, and the chest and abdomen are } \\
\text { notched }\end{array}$ & Negative \\
\hline 10 & 28 & $27^{+4}$ & $\mathrm{HL}<-4.0 \mathrm{SD}, \mathrm{FL}<-4.0 \mathrm{SD} \otimes$ femur slightly curved & Negative \\
\hline 11 & 31 & $26^{+1}$ & Fetal nasal and jaw abnormalities & Positive \\
\hline 12 & 18 & $25^{+3}$ & BPD>+2.0SD;fetal bilateral ventricular widening & Negative \\
\hline 13 & 27 & $30^{+6}$ & $\mathrm{FL}<-3.4 \mathrm{SD}, \mathrm{HL}<-2.8 \mathrm{SD}$ & Negative \\
\hline 14 & 26 & 31 & $\mathrm{BPD}>+2.0 \mathrm{SD}, \mathrm{FL}<-4.0 \mathrm{SD}, \mathrm{HL}<-4.0 \mathrm{SD}$ & Positive \\
\hline 15 & 30 & 29 & The fetus is greater than 3 weeks of the gestational week, and the femurs are slightly curved & Negative \\
\hline 16 & 28 & $25^{+2}$ & $\mathrm{FL}<-2.0 \mathrm{SD}, \mathrm{HL}<-2.0 \mathrm{SD}$;both femurs are slightly curved & Positive \\
\hline 17 & 34 & $26^{+5}$ & FL $<-3.9 S D, H L<-4.3 S D$; uneven ossification of some vertebral bodies & Positive \\
\hline 18 & 27 & $27^{+4}$ & $\mathrm{FL}<-3.0 \mathrm{SD}, \mathrm{HL}<-3.0 \mathrm{SD}$ & Negative \\
\hline 19 & 28 & $27^{+5}$ & $\mathrm{BPD}>+2.0 \mathrm{SD}, \mathrm{FL}<-4.0 \mathrm{SD}, \mathrm{HL}<-4.0 \mathrm{SD}$ & Positive \\
\hline 20 & 26 & 27 & $\mathrm{FL}<-2.0 \mathrm{SD}, \mathrm{HL}<-2.0 \mathrm{SD}$ & Negative \\
\hline 21 & 29 & $18^{+5}$ & Fetal limbs slightly curved & Positive \\
\hline 22 & 16 & 20 & $\mathrm{FL}<-4.9 \mathrm{SD}, \mathrm{HL}<-4.7 \mathrm{SD}$ & Negative \\
\hline 23 & 32 & 32 & Fetal limbs are short & Positive \\
\hline 24 & 25 & $30^{+2}$ & FL $<-3.0 S D$, Single umbilical artery & Positive \\
\hline 25 & 23 & 27 & $H L<-2.6 S D, H C<-2.8 S D$ & Positive \\
\hline 26 & 37 & $25^{+5}$ & $\mathrm{AC}<-2.0 \mathrm{SD}, \mathrm{HL}<-2.0 \mathrm{SD}, \mathrm{FL}<-2.0 \mathrm{SD}$ & Negative \\
\hline 27 & 20 & 31 & $\mathrm{FL}<-3.12 \mathrm{SD}, \mathrm{HL}<-3.12 \mathrm{SD}$ & Negative \\
\hline 28 & 29 & $24^{+6}$ & Short limb development & Positive \\
\hline 29 & 25 & $30^{+6}$ & $\mathrm{HL}<-2.9 S \mathrm{SD}, \mathrm{FL}<-2.9 \mathrm{SD}$ & Negative \\
\hline 30 & 32 & 25 & $\mathrm{FL}<-6.0 \mathrm{SD}$ & Positive \\
\hline 31 & 28 & 28 & $\mathrm{FL}<-2.7 \mathrm{SD}, \mathrm{HL}<-3.9 \mathrm{SD}$ & Negative \\
\hline 32 & 33 & 32 & $\mathrm{BPD}>+3.7 \mathrm{SD}, \mathrm{HC}>+2.8 \mathrm{SD}, \mathrm{AC}>+3.3 \mathrm{SD}$ & Negative \\
\hline 33 & 25 & $27^{+2}$ & $\mathrm{FL}<-3.0 \mathrm{SD}, \mathrm{HL}<-3.0 \mathrm{SD}$ & Negative \\
\hline 34 & 30 & $26^{+4}$ & BPD>+5.0SD, & Negative \\
\hline 35 & 25 & $23^{+4}$ & Fetal short femur & Negative \\
\hline 36 & 27 & 26 & Split vertebra of fetal half vertebra, bilateral valgus, abnormal posture of both feet and hands & Negative \\
\hline 37 & 30 & $33^{+4}$ & $\mathrm{FL}<-2.0 \mathrm{SD}$ & Negative \\
\hline 38 & 26 & $35^{+3}$ & BPD $<-5.0 S D$ & Negative \\
\hline
\end{tabular}




\begin{tabular}{|c|c|c|c|c|}
\hline 39 & 23 & $27^{+4}$ & $\mathrm{BPD}<-3.0 \mathrm{SD}, \mathrm{HC}<-2.0 \mathrm{SD}$ & Negative \\
\hline 40 & 30 & $33^{+3}$ & $\mathrm{FL}<-3.0 \mathrm{SD}, \mathrm{HL}<-2.0 \mathrm{SD}$ & Positive \\
\hline 41 & 30 & $26^{+2}$ & Bilateral foot varus & Negative \\
\hline 42 & 31 & 31 & $B P D<-2.75 S D$ & Negative \\
\hline 43 & 28 & 30 & $\mathrm{HC}>+2.5 \mathrm{SD}$ & Negative \\
\hline 44 & 26 & 27 & $\mathrm{HC}<-2.0 \mathrm{SD}, \mathrm{AC}<-2.0 \mathrm{SD}, \mathrm{HL}<-2.0 \mathrm{SD}, \mathrm{FL}<-3.0 \mathrm{SD}$ & Negative \\
\hline 45 & 38 & 26 & $\mathrm{FL}<-2.0 \mathrm{SD}, \mathrm{HL}<-2.0 \mathrm{SD}$, nose bones not shown & Positive \\
\hline 46 & 29 & $23^{+2}$ & Multi-finger & Negative \\
\hline 47 & 31 & 24 & Right foot inversion & Negative \\
\hline 48 & 34 & 33 & $H L<-2.9 S D, F L<-3.5 S D$ & Negative \\
\hline 49 & 27 & $23^{+6}$ & Bilateral foot varus,cervical thoracic spine bends backward & Positive \\
\hline 50 & 23 & $19^{+1}$ & FL $<-3.0 S D$, The femur is slightly curved and the long bone is dysplastic & Positive \\
\hline 51 & 27 & $22^{+5}$ & $\mathrm{FL}<-4.0 \mathrm{SD}, \mathrm{HL}<-3.7 \mathrm{SD}$ & Negative \\
\hline 52 & 35 & $30^{+5}$ & $\mathrm{BPD}<-3.0 \mathrm{SD}, \mathrm{HC}<-2.0 \mathrm{SD}$ & Negative \\
\hline 53 & 30 & $25^{+5}$ & Multiple vertebral ossification & Negative \\
\hline 54 & 33 & $26^{+3}$ & $\begin{array}{l}\text { Skull halo is not regular, bilateral temporal bones are slightly } \\
\text { depressed,HC<-2.0SD,AC<-2.0SD,FL }<-3.0 S D, H L<-3.0 S D\end{array}$ & Negative \\
\hline 55 & 26 & 25 & Dysplasia of fourth and fifth toes of right foot & Negative \\
\hline 56 & 28 & $25^{+5}$ & $\mathrm{FL}<-3.0 \mathrm{SD}, \mathrm{HL}<-2.9 \mathrm{SD}$ & Negative \\
\hline 57 & 26 & 33 & $\mathrm{AC}<-2.4 \mathrm{SD}, \mathrm{FL}<-3.9 \mathrm{SD}, \mathrm{HL}<-4.1 \mathrm{SD}$ & Negative \\
\hline 58 & 27 & 27 & $\mathrm{FL}<-4.0 \mathrm{SD}$ & Negative \\
\hline 59 & 29 & 32 & $\mathrm{BPD}<-4.0 \mathrm{SD}, \mathrm{HC}<-3.0 \mathrm{SD}, \mathrm{AC}<-2.0 \mathrm{SD}, \mathrm{FL}<-2.0 \mathrm{SD}$ & Negative \\
\hline 60 & 31 & $23^{+1}$ & $\begin{array}{l}\text { Nose root is flat, nose frontal angle is enlarged, nose and lip angles are acute, nose tip is drooping, upper } \\
\text { alveolar bone curvature is abnormal }\end{array}$ & Negative \\
\hline 61 & 32 & $26^{+3}$ & Spinal cone low & Negative \\
\hline 62 & 31 & $18^{+2}$ & $\mathrm{BPD}<-3.3 \mathrm{SD}, \mathrm{FL}<-5.0 \mathrm{SD}$ & Negative \\
\hline 63 & 27 & $30^{+4}$ & $\mathrm{FL}<-3.0 \mathrm{SD}, \mathrm{HL}<-3.0 \mathrm{SD}$ & Negative \\
\hline 64 & 42 & 27 & $\mathrm{BPD}<-2.5 \mathrm{SD}, \mathrm{FL}<-4.0 \mathrm{SD}$ & Negative \\
\hline 65 & 15 & $24^{+5}$ & Multi-finger & Negative \\
\hline 66 & 28 & 23 & Multi-finger & Negative \\
\hline 67 & 23 & 23 & $\begin{array}{l}\mathrm{HC}<-2.0 \mathrm{SD}, \mathrm{HL}<-8.0 \mathrm{SD}, \mathrm{FL}<-5.0 \mathrm{SD} ; \mathrm{Abnormal} \text { ossification of both humerus and femur, poor ossification of } \\
\text { cervical vertebra }\end{array}$ & Positive \\
\hline 68 & 30 & $24^{+3}$ & Bilateral foot varus & Negative \\
\hline 69 & 36 & $23^{+5}$ & $\mathrm{BPD}<-2.0 \mathrm{SD}, \mathrm{AC}<-3.0 \mathrm{SD}, \mathrm{FL}<-5.0 \mathrm{SD}$ & Negative \\
\hline 70 & 29 & 23 & $\mathrm{FL}<-8.0 \mathrm{SD}, \mathrm{HC}<-4.9 \mathrm{SD}$ & Negative \\
\hline 71 & 29 & 23 & L1 half cone, T11 split vertebra & Negative \\
\hline 72 & 29 & $30^{+3}$ & $\mathrm{FL}<-4.0 \mathrm{SD}, \mathrm{HL}<-2.0 \mathrm{SD}$ & Positive \\
\hline 73 & 30 & 26 & Nasal bone dysplasia & Positive \\
\hline 74 & 29 & $31^{+1}$ & Fusion vertebra & Negative \\
\hline 75 & 31 & 28 & $\mathrm{BPD}<-3.2 \mathrm{SD}, \mathrm{HC}<-2.5 \mathrm{SD}$ & Negative \\
\hline 76 & 29 & $24^{+3}$ & Bilateral foot varus,Abnormal hand posture & Positive \\
\hline 77 & 21 & 27 & Fingers merge, toes merge, temporal depression.Pregnant women also have finger merge and toe merge & Positive \\
\hline 78 & 41 & 31 & $\mathrm{FL}<-3.69 \mathrm{SD}$ & Negative \\
\hline
\end{tabular}


Table.2 Correlation between abnormal results of FGFR3 gene sequenced by WESand clinical phenotypes by ultrasound

\begin{tabular}{|c|c|c|c|c|c|c|c|}
\hline ID & Phenotype by ultrasound & $\begin{array}{l}\text { Reference } \\
\text { sequence }\end{array}$ & $\begin{array}{l}\text { Sequencing results by } \\
\text { clinical WES }\end{array}$ & Geneticsubregion & Heterogeneity & ChromosomalLoci & $\mathrm{Mu}$ \\
\hline 7 & $\begin{array}{l}\mathrm{HL}<-5.9 S D, F L<-5.9 S D \otimes \\
\text { metaphyseal enlargement }\end{array}$ & NM_000142.4 & FGFR3:c.1138G>A(p.G380R) & Exon9 & Het & $\begin{array}{l}\text { chr4:1806119- } \\
1806119\end{array}$ & Pa1 \\
\hline 14 & BPD $>+2.0 S D, F L<-4.0 S D, H L<-4.0 S D$ & NM_000142.4 & FGFR3:c.1138G>A(p.G380R) & Exon9 & Het & $\begin{array}{l}\text { chr4:1806119- } \\
1806119\end{array}$ & $\mathrm{~Pa} 1$ \\
\hline 19 & BPD $>+2.0 S D, F L<-4.0 S D, H L<-4.0 S D$ & NM_000142.4 & FGFR3:c.1620C>A(p.N540K) & Exon12 & Het & $\begin{array}{l}\text { chr4:1807371- } \\
1807371\end{array}$ & Pa1 \\
\hline 23 & Fetal limbs are short & NM_000142.4 & FGFR3:c.1138G>A(p.G380R) & Exon9 & Het & $\begin{array}{l}\text { chr4:1806119- } \\
1806119\end{array}$ & $\mathrm{~Pa} 1$ \\
\hline 30 & $\mathrm{FL}<-6.0 \mathrm{SD}$ & NM_000142.4 & FGFR3:c.1138G>A(p.G380R) & Exon9 & Het & $\begin{array}{l}\text { chr4:1806119- } \\
1806119\end{array}$ & Pa1 \\
\hline 72 & $\mathrm{FL}<-4.0 \mathrm{SD}, \mathrm{HL}<-2.0 \mathrm{SD}$ & NM_000142.4 & FGFR3:c.1138G>A(p.G380R) & Exon9 & Het & $\begin{array}{l}\text { chr4:1806119- } \\
1806119\end{array}$ & Pa1 \\
\hline 79 & $\mathrm{HC}>+2.4 S \mathrm{~S}, \mathrm{AC}<-0.34 \mathrm{SD}, \mathrm{FL}<-4.2 \mathrm{SD}$ & NM_000142.4 & FGFR3:c.1138G>A(p.G380R) & Exon9 & Het & $\begin{array}{l}\text { chr4:1806119- } \\
1806119\end{array}$ & $\mathrm{~Pa} 1$ \\
\hline
\end{tabular}

Table.3 Correlation between abnormal results of COL $1 A 1$ gene sequenced by WESand clinical phenotypes by ultrasound

\begin{tabular}{|c|c|c|c|c|c|c|c|}
\hline ID & $\begin{array}{l}\text { Phenotype by } \\
\text { ultrasound }\end{array}$ & $\begin{array}{l}\text { Reference } \\
\text { sequence }\end{array}$ & $\begin{array}{l}\text { Sequencing results by clinical } \\
\text { WES }\end{array}$ & Geneticsubregion & Heterogeneity & ChromosomalLoci & Mutationtyp€ \\
\hline 2 & $\begin{array}{l}\mathrm{HL}<-3.2 \mathrm{SD}, \mathrm{FL}<-2.1 \mathrm{SD} ; \\
\text { both femurs are } \\
\text { slightly curved }\end{array}$ & NM_000088.3 & COL 1A1:c.896G>A(p.G299D) & Exon13 & Het & $\begin{array}{l}\text { chr17:48273852- } \\
48273852\end{array}$ & Pathogenic \\
\hline 16 & $\begin{array}{l}\mathrm{FL}<-2.0 \mathrm{SD}, \mathrm{HL}<-2.0 \mathrm{SD} \text {; } \\
\text { both femurs are } \\
\text { slightly curved }\end{array}$ & NM_000088.3 & COL1A1:c.3235G>A(p.G1079S) & Exon44 & Het & $\begin{array}{l}\text { chr17:48265483- } \\
48265483\end{array}$ & $\begin{array}{l}\text { Likely } \\
\text { pathogenic }\end{array}$ \\
\hline 21 & $\begin{array}{l}\text { Fetal limbs slightly } \\
\text { curved }\end{array}$ & NM_000088.3 & COL1A1:c.1301G>A(p.G434D) & Exon20 & Het & $\begin{array}{l}\text { chr17:48272460- } \\
48272460\end{array}$ & Pathogenic \\
\hline
\end{tabular}

Table.4 Correlation between abnormal results of othergene sequenced by WESand clinical phenotypes by ultrasound 


\begin{tabular}{|c|c|c|c|c|c|c|}
\hline ID & Phenotype by ultrasound & $\begin{array}{l}\text { Reference } \\
\text { sequence }\end{array}$ & $\begin{array}{l}\text { Sequencing results by clinical } \\
\text { WES }\end{array}$ & Geneticsubregion & Heterogeneity & Chromosc \\
\hline 6 & $\begin{array}{l}\text { Poor skull development, nasal bones not } \\
\text { shown, poor ossification of the lumbar } \\
\text { vertebral body of the spine, poor } \\
\text { ossification of the sacral caudal vertebral } \\
\text { arch and vertebral body }\end{array}$ & NM_001024630.3 & RUNX2:c.931_946del(p.T311fs) & Exon7 & Het & $\begin{array}{l}\text { chr6:4548 } \\
4548006 \text { ؟ }\end{array}$ \\
\hline 11 & Fetal nasal and jaw abnormalities & NM_000047.2 & ARSE:c.331C>T(p.R111C) & Exon5 & Hemi & $\begin{array}{l}\text { chrX:2871 } \\
2871283\end{array}$ \\
\hline 17 & $\begin{array}{l}\mathrm{FL}<-3.9 \mathrm{SD}, \mathrm{HL}<-4.3 \mathrm{SD} \text {; uneven ossification } \\
\text { of some vertebral bodies }\end{array}$ & NM_001844.4 & COL2A1:c.1420-2A>G & Intron22 & Het & $\begin{array}{l}\text { chr12:483 } \\
4838022 \varepsilon\end{array}$ \\
\hline 25 & $H L<-2.6 S D, H C<-2.8 S D$ & NM_004247.3 & EFTUD2:c.1705C>T(p.R569X) & Exon 17 & Het & $\begin{array}{l}\text { chr17:429 } \\
42937814\end{array}$ \\
\hline 28 & Short limb development & NM_001844.4 & COL2A1:c.1924G>A(p.G642R) & Exon29 & Het & $\begin{array}{l}\text { chr12:483 } \\
48377887\end{array}$ \\
\hline 45 & $\begin{array}{l}\mathrm{FL}<-2.0 \mathrm{SD}, \mathrm{HL}<-2.0 \mathrm{SD} \text {, nose bones not } \\
\text { shown }\end{array}$ & $\begin{array}{l}\text { NM_001024630 } \\
.3\end{array}$ & RUNX2:c.568C>T(p.R190W) & Exon9 & Het & $\begin{array}{l}\text { chr6:4539 } \\
45399744\end{array}$ \\
\hline \multirow[t]{2}{*}{49} & $\begin{array}{l}\text { Bilateral foot varus,cervical thoracic spine } \\
\text { bends backward }\end{array}$ & NM_000334.4 & SCN4A:c.4361G>A(p.R1454Q) & Exon24 & Het & $\begin{array}{l}\text { chr17:620 } \\
62019281\end{array}$ \\
\hline & & NM_000334.4 & SCN4A:c.481G>A(p.E161K) & Exon3 & Het & $\begin{array}{l}\text { chr17:620 } \\
62049497\end{array}$ \\
\hline 50 & $\begin{array}{l}\mathrm{FL}<-3.0 \mathrm{SD} \text {, The femur is slightly curved } \\
\text { and the long bone is dysplastic }\end{array}$ & NM_000089.3 & COL 1A2:c.2261G>T(p.G754V) & Exon37 & Het & $\begin{array}{l}\text { chr7:9404 } \\
94049926\end{array}$ \\
\hline \multirow[t]{2}{*}{67} & $\begin{array}{l}\mathrm{HC}<-2.0 \mathrm{SD}, \mathrm{HL}<-8.0 \mathrm{SD}, \mathrm{FL}<-5.0 \mathrm{SD} \text {; } \mathrm{Abnormal} \\
\text { ossification of both humerus and femur, } \\
\text { poor ossification of cervical vertebra }\end{array}$ & NM_000288.3 & PEX7:c.283T>G(p.W95G) & Exon3 & Het & $\begin{array}{l}\text { chr6:1371 } \\
13714755\end{array}$ \\
\hline & & NM_000288.3 & PEX7:c.408dup(p.V137Cfs*16) & Exon4 & Het & $\begin{array}{l}\text { chr6:1371 } \\
13716682\end{array}$ \\
\hline \multirow[t]{2}{*}{76} & $\begin{array}{l}\text { Bilateral foot varus,Abnormal hand } \\
\text { posture, }\end{array}$ & NM_001271208.1 & $N E B: c .1569+5 G>A$ & Exon17 & Het & $\begin{array}{l}\text { chr2:1525 } \\
15255314\end{array}$ \\
\hline & & NM_001271208.1 & NEB:c. $2278 \mathrm{C}>\mathrm{T}(\mathrm{p} . \mathrm{Q} 760 *)$ & Exon24 & Het & $\begin{array}{l}\text { chr2:1525 } \\
15254727\end{array}$ \\
\hline 77 & $\begin{array}{l}\text { Fingers merge, toes merge, temporal } \\
\text { depression,.Pregnant women also have } \\
\text { finger merge and toe merge }\end{array}$ & NM_000141.4 & FGFR2:c.755C>G(p.S252W) & Exon7 & Het & $\begin{array}{l}\text { chr10:123 } \\
12327967\end{array}$ \\
\hline
\end{tabular}

Table.5 Correlation between abnormal results of copy number variation (CNV) sequenced by WESand clinical phenotypes by ultrasound 


\begin{tabular}{|c|c|c|c|c|c|c|}
\hline ID & $\begin{array}{l}\text { Phenotype by } \\
\text { ultrasound }\end{array}$ & Chromosome & $\begin{array}{l}\text { Micro- } \\
\text { deletion/duplication } \\
\text { results and } \\
\text { significance }\end{array}$ & Size(bp) & Related genes & Related diseases \\
\hline 5 & $\begin{array}{l}\text { Curved fetal } \\
\text { osteogenesis }\end{array}$ & ND & $\begin{array}{l}\text { 1q21.1-q21.2 } \\
\text { deletion, } \\
\text { chr1:146631138- } \\
147415663\end{array}$ & $0.78 \mathrm{M}$ & GJA5;GJA8 & $\begin{array}{l}\text { 1q21.1 recurrentmicrodeletion } \\
\text { locus forneurodevelopmental }\end{array}$ \\
\hline 24 & $\begin{array}{l}\text { FL<-3.0SD, Single } \\
\text { umbilical artery }\end{array}$ & ND & $\begin{array}{l}\text { 2q35-q36.1 } \\
\text { deletion, } \\
\text { chr2:219247086- } \\
222436973\end{array}$ & $3.19 \mathrm{M}$ & EPHA4 & $2 q 35-q 36.1$ deletion \\
\hline 40 & $\mathrm{FL}<-3.0 \mathrm{SD}, \mathrm{HL}<-2.0 \mathrm{SD}$ & ND & $\begin{array}{l}\text { Xp22.33-p21.2 } \\
\text { deletion, } \\
\text { chrX:2700101- } \\
30327485\end{array}$ & $27.63 \mathrm{M}$ & $\begin{array}{l}\text { ARSE;NLGN4X;STS;ANOS1;GPR143; } \\
\text { CLCN4;MID1;HCCS;AMELX;FRMPD4; } \\
\text { TRAPPC2;OFD1;FANCB;PIGA;AP1S2; } \\
\text { NHS;CDKL5;RS1;PHKA2;ADGRG2; } \\
\text { PDHA1;RPS6KA3;CNKSR2;SMPX; } \\
\text { MBTPS2;SMS;PHEX;PTCHD1;KLHL15; } \\
\text { EIF2S3;PDK3;POLA1;ARX;IL1RAPL }\end{array}$ & ND \\
\hline & & ND & $\begin{array}{l}\text { Xq21.31- q28 } \\
\text { deletion, } \\
\text { chrX:88008410- } \\
154774942\end{array}$ & $66.77 \mathrm{M}$ & $\begin{array}{l}\text { DIAPH2;PCDH19;SRPX2;TIMM8A; } \\
\text { BTK;GLA;HNRNPH2;GPRASP2; } \\
\text { PLP1;SERPINA7;PIH1D3;PRPS1; } \\
\text { MID2;COL4A6;COL4A5;ACSL4;AMMECR1 } \\
; \\
\text { CHRDL 1;PAK3;DCX;ALG13;PLS3;CXOrf56; } \\
\text { UBE2A;UPF3B;RNF113A;NDUFA1;LAMP2; } \\
\text { CUL4B;C1GALT1C1;GLUD2 }\end{array}$ & ND \\
\hline 73 & $\begin{array}{l}\text { Nasal bone } \\
\text { dysplasia }\end{array}$ & Trisomy 21 & $\begin{array}{l}\text { 21q11.2- } \\
\text { q22.3Duplication, } \\
\text { chr21:14982544- } \\
48084291\end{array}$ & $33.1 \mathrm{M}$ & ND & Down's Syndrome \\
\hline
\end{tabular}

\section{Discussion}

By studying the WES test results of 79 pregnant women from the third affiliated hospital of Zhengzhou University from August 2018 to April 2020 , the overall detection rate of skeletal abnormalities was $31.6 \%$. This is higher than the previously reported detection rate (15.4\%[7] and $24 \%[5])$. This may be due to our stricter requirements for the ultrasound test results of the enrolled cases. Interestingly, we find that for different types of skeletal abnormalities, the detection rate through WES varies greatly. Among them, WES has a higher detection rate for short limbs. Especially when $\mathrm{FL}<-4.0 \mathrm{SD}$ or $\mathrm{HL}<-4.0 \mathrm{SD}$, the detection rate can rise to $41.6 \%$. However, when the fetal has short limbs with other bone abnormal phenotypes, the detection rate will be higher. For example, when the limbs are short with bone curved, the WES detection rate can reach $100 \%$; when the limbs are short with nasal bone dysplasia, WES detection rate can reach $80 \%$. On the contrary, if the phenotype of short limbs is not detected by ultrasound testing and only other skeletal abnormal phenotypes exists, such as only fetal hand or foot deformities, the WES detection rate will be very low, only $11.1 \%$. It may be that achondroplasia is the cause for the most of skeletal abnormalities [8], which is also the most common form of inherited disproportionate short stature[9]. In our study, through ultrasound, 46 of 79 fetal skeletal abnormalities had clinical manifestations of short limbs, accounting for $58.2 \%$, consistent with previous reports[10]. This shows that the diagnosis of achondroplasia is the key to the diagnosis of fetal skeletal abnormalities.

Through our research, we find that for the diagnosis of fetal FGFR3-related achondroplasia, the WES detection rate is highly correlated with the results of ultrasound testing of the fetal limb shortness and severity.Through our research, we find that the diagnosis of fetal FGFR3-related achondroplasia[11] accounts for the highest proportion of all skeletal abnormalities, reaching $28 \%$. Almost all the ultrasound test results of fetal achondroplasia have been detected with $\mathrm{FL}<-4.0 \mathrm{SD}$ or $\mathrm{HL}<-4.0 \mathrm{SD}$, suggesting that $\mathrm{FL}<-4.0 \mathrm{SD}$ or $\mathrm{HL}<-4.0 \mathrm{SD}$ is the most critical basis for the diagnosis of achondroplasia by fetal ultrasound testing.At the same time, the detection of macrocephaly through ultrasound is also an important evidence for the diagnosis of fetal achondroplasia.Among the 7 cases of FGFR3-related achondroplasia, 6 cases were caused by FGFR3 gene c.1138G>A mutation. FGFR3 gene c.1138G>A mutation is the most common mutation in FGFR3-related achondroplasia, which accounts for more than $99 \%$ of all FGFR3-related achondroplasia together with FGFR3 gene c.1138G>C mutation[12, 13].At this point, our research is consistent with these reports.In addition, we have also detected a case of FGFR3 gene c.1620C>A mutation through WES, which is related to hypochondroplasia[14].FGFR3 gene c.1620C>A mutation has been reported extensively[15] and is 
considered to account for 50\%-76\% of FGFR3-related hypochondroplasia[16].Therefore, FGFR3 gene c.1138G>A mutation and c.1620C>A mutation may be the two most common causes of skeletal abnormalities in the fetal period and require special clinical attention.

Osteogenesis imperfecta caused by the COL $1 A 1$ gene is also a major cause of fetal skeletal dysplasia. The main feature of Osteogenesis imperfecta is multiple fractures usually caused by minor trauma[17, 18]. This may be manifested by the limbs being curved by ultrasound testing during the prenatal period.In our study, osteogenesis imperfecta due to COL1A1 gene mutation was detected in the presence of limbs slightly curved through ultrasound in the fetal period,which suggests that the presence of this phenotype during the fetal period may be highly correlated with COL1A1-related osteogenesis imperfecta. In addition, COL 1A1 gene c.896G>A mutation and c.1301G>A mutation found in our research have not been reported in other studies before, which broadens the clinical understanding of this gene mutation. $C O L 1 A 1$ gene $c .3235 \mathrm{G}>\mathrm{A}$ mutation, according to previous reports, shows that it has a highly variable phenotype in the family, and family members with this gene mutation can only show signs of disease without fractures[19, 20]. This may explain why in our study, the husband of the pregnant woman also has the heterozygous mutation of the gene but the phenotype was not abnormal.

In our study, 2 cases of cleidocranial dysplasia related to RUNX2 gene mutation were detected, which were c.931_946del mutation and c.568C >T mutation. The main clinical features of cleidocranial dysplasia include persistently open skull sutures with bulging calvaria, hypoplasia or aplasia of the clavicles permitting abnormal facility in apposing the shoulders, wide pubic symphysis, short middle phalanx of the fifth fingers, dental anomalies, and often vertebral malformation[21]. It has been pointed out in previous reports that cleidocranial dysplasia can also be related to the phenotype of nasal bone loss[21], which is consistent with our case study.In our study, some other genetic variations or chromosomal aneuploidies related to the nasal bone loss phenotype are also found, such as the ARSE gene c.331C >T variation and trisomy 21.ARSE gene mutation is related to X-linked recessive chondrodysplasia punctata, which is manifested as nasal dysplasia and distal phalanx dysplasia[22], which is consistent with the phenotype observed during fetal ultrasound testing. Trisomy 21 is the most frequent form of mental retardation caused by a microscopically demonstrable chromosomal aberration, is characterized by well-defined and distinctive phenotypic features and natural history[23]. It has been reported that nasal bone dysplasia is a common detected phenotype of fetal trisomy 21[24], which is consistent with the phenotype of a trisomy 21 case found in our study. Therefore, in the diagnosis of fetal skeletal abnormalities, nasal bone dysplasia may be another typical indication of ultrasound abnormality in addition to short limbs.

In our study, some gene mutations related to skeletal abnormalities related to hand and foot abnormalities are also detected. For example, SCN4A gene c.4361G >A mutation[25], NEB gene c.1569+5G>A and c.2278C > T compound heterozygous mutation[26], FGFR2 gene c.755C>G mutation[27]. Most of the diseases related to these gene mutations mainly affect the function of the muscular system as the main cause[28, 29], and their detection rate in fetal skeletal abnormalities is low (only FGFR2 gene c.755C>G mutation), but their detection can play an important guiding role in clinical diagnosis and treatment.

\section{Conclusions}

In conclusions, our research shows that the application of whole exome sequencing technology can significantly improve the systemic prenatal diagnosis of skeletal abnormalities, and according to the different types of ultrasound detection results, WES has different detection rates for various skeletal abnormalities. Through our research, it is shown that fetal short limbs are the best detection targets for WES to detect skeletal abnormalities. In addition, the fetal limbs curved and nasal bone dysplasia are also important clinical phenotypes that suggest genetic variation-related skeletal abnormalities. However, the genetic basis of bone diseases is still unknown in other respects, indicating that new genes or non-genetic factors may cause these diseases.

\section{Materials And Methods}

\section{Pregnant women Enrollment!specimens and DNA preparation}

We selected a total of 79 pregnant women from the third affiliated hospital of Zhengzhou University, China, from August 2018 to April 2020 , who had undergone whole exome sequencing due to fetal ultrasound suggesting skeletal dysplasia. Sample entry requirements: pregnant women between 18-36 weeks of gestation, ultrasound examination shows femur length <-2SD or humerus length <-2SD or other skeletal development abnormalities: such as nasal bone dysplasia, limb bone deformity, skull or clavicle development Exception etc. We performed amniocentesis on the enrolled pregnant women for subsequent DNA extraction.Afterwards, we used QIAGEN 69504 blood and tissue DNA extraction kit for DNA extraction from amniotic fluid, peripheral blood of pregnant women and their husbands.

\section{Whole exome sequencing(WES) and CNV analysis}

The genomic DNA of amniotic fluid was broken into random fragments and purified, and whole genome exome capture was performed for sequencing library preparation. The Illumina Hiseq XTen sequencer was used to perform double-ended high-throughput sequencing with a length of $150 \mathrm{bp}$. The raw data obtained by the sequencing were quality-controlled, and the basic data were analyzed and filtered to remove the linker sequence and repeat sequence. Data were analyzed using the Anno variant site detection system, the XYGeneRanger variant site annotation interpretation system.

\section{Variant annotation and interpretation}

The pathogenicity assessment and data interpretation rules of mutation are based on the guidelines of the American College of Medical Genetics and Genomics (ACMG) [30]. By querying 1000genomes, ExAC and gnomAD, we excluded gene mutations whose frequency of the population are more than $1 \%$ and removed non-functional mutation sites (such as synonymous mutations, non-coding region mutations, etc.). We performed gene function prediction (using software such as SIFT, Polyphen2, CADD, etc.) and clinical symptoms comparison. At last, we searched related disease databases and related references, and finally found candidate gene mutation sites for family verification. The variant annotation databases which were used include Human Genome hg19/GRCh37, 
RefSeq, dbSNP, 1000 Genomes phase3, ExAC, and gnomAD and the interpretation databases which were used include DGV, DECIPHER, OMIM, UCSC, ClinVar, HGMD and PubMed.

\section{Family verification by sanger sequencing}

Genetic modification of the mutations was performed on the fetus, pregnant women and their husbands. According to the exome of the mutation site, the primers needed for sequencing the synthetic DNA fragments were designed, and the DNA of the fetus, pregnant women and their husbands were PCRamplified. Method of sequencing was performed by Sanger sequencing and the sequencing results were compared with the results of whole exome sequencing.

\section{Declarations}

\section{Acknowledgements}

We thank the the Third Affiliated Hospital of Zhengzhou University for their interest and cooperation.

\section{Funding}

This work was supported by Henan Medical Science and Technology Fund (2018020176) and Zhengzhou collaborative Innovation Major Project (18XTZX12009)

\section{Author information}

\section{Affiliations}

Department of clinical laboratory, Third affiliated hospital of Zhengzhou University, Zhengzhou, 450052, China

Yuan Tian, Erfeng Yuan, Jinshuang Gao, Yaqing Guo, Haiyang Yu , Linlin Zhang, and Enwu Yuan

Department of medical imaging science, Third affiliated hospital of Zhengzhou University, Zhengzhou, 450052, China.

Xiao'an Zhang and Xin Zhao

Department of obstetrics and gynecology, Third affiliated hospital of Zhengzhou University, Zhengzhou,450052, China.

Shihong Cui

\section{Author contribution}

Yuan Tian, Linlin Zhang and Xiao'an Zhang participated in Studying concept and design. All authors participated in acquisition ,analysis and interpretation of data.

Yuan Tian and Haiyang Yu participated in Drafting of the manuscript. All authors participated in Critical revision of the manuscript for important intellectual content.

Erfeng Yuan, Jinshuang Gao and Yaqing Guo participated in Data Analysis

\section{Corresponding authors}

Correspondence to Linlin Zhang or Xiao'an Zhang.

\section{Ethics declarations}

\section{Ethics approval and consent to participate}

Experiment on human subjects was approved by the Third Affiliated Hospital of Zhengzhou University, Zhengzhou, China.

Informed consent for participation to this study was obtained from all individuals.

\section{Consent for publication}

Publication of data was informed consent to all individuals involved in this study.

\section{Competing interests}

The authors declare no conflict of interests.

\section{Data Availability Statements}

The data that support the findings of this study are available from the corresponding author upon reasonable request. 


\section{References}

1. Grannum PA, Hobbins JC. Prenatal diagnosis of fetal skeletal dysplasias. Semin Perinatol. 1983; 7(2): 125.

2. Liu Y, Wang L, Yang Y, Liang Y, Wu Q. Prenatal diagnosis of fetal skeletal dysplasia using targeted next-generation sequencing: an analysis of 30 cases. Diagn Pathol. 2019; 14(1): 76.

3. Yang K, Shen M, Yan Y, Tan Y, Tian Y. Genetic analysis in fetal skeletal dysplasias by trio whole-exome sequencing. Biomed Res Int. 2019; 2019(2): 1-8.

4. Tang J, Zhou C, Shi H, Mo Y, Luo L. Prenatal diagnosis of skeletal dysplasias using whole exome sequencing in china. Clin Chim Acta. $2020 ; 507$

5. Petrovski S, Aggarwal V, Giordano JL, Stosic M, Wou K, Bier Let al. Whole-exome sequencing in the evaluation of fetal structural anomalies: a prospective cohort study. Lancet. 2019; 393(10173): 758-67.

6. Warman ML, Cormier-Daire V, Hall C, Krakow D, Lachman R, Lemerrer Met al. Nosology and classification of genetic skeletal disorders: 2010 revision. Am J Med Genet a. 2011; 155(5): 943-68.

7. Lord J, McMullan DJ, Eberhardt RY, Rinck G, Wilson E. Prenatal exome sequencing analysis in fetal structural anomalies detected by ultrasonography (page): a cohort study. Lancet. 2019; 393(10173): 747-57.

8. Pauli RM. Achondroplasia: a comprehensive clinical review. Orphanet J Rare Dis. 2019; 14(1)

9. Waller DK, Correa A, Vo TM, Wang Y, Hobbs C, Langlois PHet al. The population-based prevalence of achondroplasia and thanatophoric dysplasia in selected regions of the us. Am J Med Genet a. 2008; 146A(18): 2385-9.doi: 10.1002/ajmg.a.32485.

10. Georgoulis G, Alexiou G, Prodromou N. Achondroplasia with synostosis of multiple sutures. Am J Med Genet a. 2011; 155(8): 1969-71.

11. Wynn J, King TM, Gambello MJ, Waller DK, Hecht JT. Mortality in achondroplasia study: a 42-year follow-up. Am J Med Genet a. 2007; 143A(21): 2502-11.

12. Xue Y, Sun A, Mekikian PB, Martin J, Wilcox WR. Fgfr3 mutation frequency in 324 cases from the international skeletal dysplasia registry. Mol Genet Genomic Med. 2014; 2(6): 497-503.

13. Foldynova-Trantirkova S, Wilcox WR, Krejci P. Sixteen years and counting: the current understanding of fibroblast growth factor receptor 3 (fgfr3) signaling in skeletal dysplasias. Hum Mutat. 2012; 33(1): 29-41.

14. Bellus GA, Mcintosh I, Smith EA, Aylsworth AS, Francomano CA. A recurrent mutation in the tyrosine kinase domain of fibroblast growth factor receptor 3 causes hypochondroplasia. Nat Genet. 1995; 10(3): 357-9.

15. Deutz-Terlouw PP, Losekoot M, Aalfs CM, Hennekam RCM, Bakker E. Asn540thr substitution in the fibroblast growth factor receptor 3 tyrosine kinase domain causing hypochondroplasia. Hum Mutat. 1998;

16. Tarja, Linnankivi, Outi, Mäkitie, Leena, Valanneet al. Neuroimaging and neurological findings in patients with hypochondroplasia andfgfr3n540k mutation. Am J Med Genet a. 2012;

17. W RD, R SJ, M P, S S. Diminished type i collagen synthesis and reduced alpha 1(i) collagen messenger rna in cultured fibroblasts from patients with dominantly inherited (type i) osteogenesis imperfecta. J Clin Invest. 1985;

18. Willing MC, Cohn DH, Byers PH. Frameshift mutation near the 3 ' end of the col1a1 gene of type i collagen predicts an elongated pro alpha 1 (i) chain and results in osteogenesis imperfecta type i. J Clin Invest. 1990; 85(1): 282-90.

19. D. MMV, M. M, P. ABV, V. S, O. RAMR, R. NVRet al. Variable expressivity of osteogenesis imperfecta in a brazilian family due to p.g1079s mutation in the col1a1 gene. Genetics \& Molecular Research. 2012; 11(3): 3246-55.

20. Kaneko H, Kitoh H, Matsuura T, Masuda A, Ito M, Mottes Met al. Hyperuricemia cosegregating with osteogenesis imperfecta is associated with a mutation in gpatch8. Hum Genet. 2011; 130(5): 671-83.

21. Pan C, Tseng Y, Lan T, Chang H. Craniofacial features of cleidocranial dysplasia. J Dent Sci. 2017: S866218991.

22. Brunetti-Pierri N, Andreucci M, Tuzzi R, Vega G, Gray G, McKeown Cet al. X-linked recessive chondrodysplasia punctata: spectrum of arylsulfatase e gene mutations and expanded clinical variability. American Journal of Medical Genetics. 2003; 117A(2): 164-8.

23. Zhu JL, Hasle H, Correa A, Schendel D, Friedman JM, Olsen Jet al. Survival among people with down syndrome: a nationwide population-based study in denmark. Genet Med. 2013; 15(1): 64-9.doi: 10.1038/gim.2012.93. 
24. De Jong Pleij EAP, Vos Fl, Ribbert LSM, Pistorius LR, Tromp E, Bilardo CM. Prenasal thickness-to-nasal bone length ratio: a strong and simple second- and third-trimester marker for trisomy 21. Ultrasound Obstet Gynecol. 2012; 39(2)

25. Gay S, Dupuis D, Faivre L, Masurelpaulet A, Labenne M, Colombani Met al. Severe neonatal non-dystrophic myotonia secondary to a novel mutation of the voltage-gated sodium channel (scn4a) gene. Am J Med Genet a. 2010; 146A(3): 380-3.

26. Lehtokari VO, Kiiski K, Sandaradura SA, Laporte J, Ettersson CW. Mutation update: the spectra of nebulin variants and associated myopathies. Hum Mutat. $2014 ; 35(12)$

27. Miraoui H, Ringe J, Häupl T. Increased efg- and pdgfa-receptor signaling by mutant fgf-receptor 2 contributes to osteoblast dysfunction in apert craniosynostosis. Hum Mol Genet. 2010; 19(9): 1678-89.

28. Coen A. C. Ottenheijm CCWG. Thin filament length dysregulation contributes to muscle weakness in nemaline myopathy patients with nebulin deficiency. Hum Mol Genet. 2009; 18: 2359-69.

29. Matthews E, Manzur AY, Sud R, Muntoni F, Hanna MG. Stridor as a neonatal presentation of skeletal muscle sodium channelopathy. Archives of Neurology. $2011 ; 68(1): 127-9$.

30. Richards S, Aziz N, Bale S, Bick D, Das S, Gastier-Foster Jet al. Standards and guidelines for the interpretation of sequence variants: a joint consensus recommendation of the american college of medical genetics and genomics and the association for molecular pathology. Genetics in Medicine Official Journal of the American College of Medical Genetics. 2015; 17(5): 405-23. 